\title{
Search for FRB and FRB-like single pulses in Parkes magnetar data
}

\author{
M. Burgay, ${ }^{1}$ P. Esposito, ${ }^{2}$ G.L. Israel, ${ }^{3}$ N. Rea,${ }^{2,4}$ A. Possenti, ${ }^{1}$ and \\ J. Sarkissian ${ }^{5}$ \\ ${ }^{1}$ INAF - Osservatorio Astronomico di Cagliari, Italy \\ email: burgay@oa-cagliari.inaf .it \\ ${ }^{2}$ UvA - Anton Pannekoek Institute, The Netherlands \\ ${ }^{3}$ INAF - Osservatorio Astronomico di Roma, Italy \\ ${ }^{4}$ CSIC-IEEC - Instituto de Ciencias del Espacio, Spain \\ ${ }^{5}$ CASS - Parkes Observatory, NSW, Australia
}

\begin{abstract}
We present the results of a search for strong single radio pulses emitted by magnetars and for FRB signals in the fields of magnetars observed at the Parkes radio telescope within the NAPA project $\mathrm{P} 626$. Unsurprisingly, given the short total observing time, no extragalacic FRB signal was found up to a DM of $3000 \mathrm{pc} / \mathrm{cm}^{3}$. Two strong pulses dispersed at the DM of the known radio magnetar J1550-5418 where found, one occurring at the same time of an X-ray burst. This result is potentially interesting in the framework of magnetar models for FRBs.
\end{abstract}

Keywords. stars: neutron, stars: pulsar: individual (J1550-5418, J1809-1943), X-rays: bursts

\section{Observations}

Radio data were taken at Parkes with various backends (AFB, DFB3, BPSR) and frequencies as part of the NAPA project P626 aimed at observing transient magnetars in $\mathrm{X}$-ray outburst to catch the onset of transient radio emission. The list of sources observed and the details of the observing setups are summarised in Table 1.

Table 1. Main characteristics of radio observations (source name, start date, length, central frequency and sampling time). In bold, data with simultaneous X-ray coverage.

\begin{tabular}{c|c|c|c|c}
\hline Source & SizeMJD start & Length (hr) & Freq $(\mathbf{G H z})$ & $\mathbf{t}_{\text {sam } p l}(\mathbf{m s})$ \\
\hline J0755-2933 & 57472.213 & 1.9 & 1.4 & 0.25 \\
$\mathrm{~J} 1550-5418$ & 54856.700 & 1.2 & 3.1 & 1.00 \\
& 54865.770 & $\mathbf{1 . 5}$ & 6.4 & 1.00 \\
$\mathrm{~J} 1809-1943$ & 54359.220 & $\mathbf{7 . 0}$ & 1.4 & 0.5 \\
$\mathrm{~J} 1833-0832$ & 55279.954 & 1.6 & 1.4 & 1.00 \\
$\mathrm{~J} 1934+2153$ & 56872.473 & 1.5 & 1.4 & 0.064 \\
& 56871.479 & 1.5 & 3.1 & 1.00 \\
\hline
\end{tabular}

For J1550-5418 and J1809-1943 (two magnetars with known transient pulsed radio emission; Camilo et al. 2007, Camilo et al. 2006), X-ray observations were taken simultaneously with XMM-Newton. Their total duration was 56 and $75 \mathrm{ks}$, respectively, for the two sources, completely covering the time-span of the radio data (in bold-face in the table above).

\section{Data analysis}

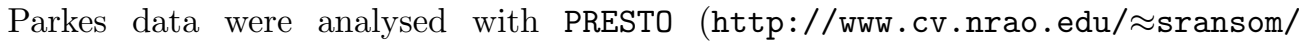
presto/); all data sets were barycentered and de-dispersed up to a DM of $3000 \mathrm{pc} / \mathrm{cm}^{3}$ 

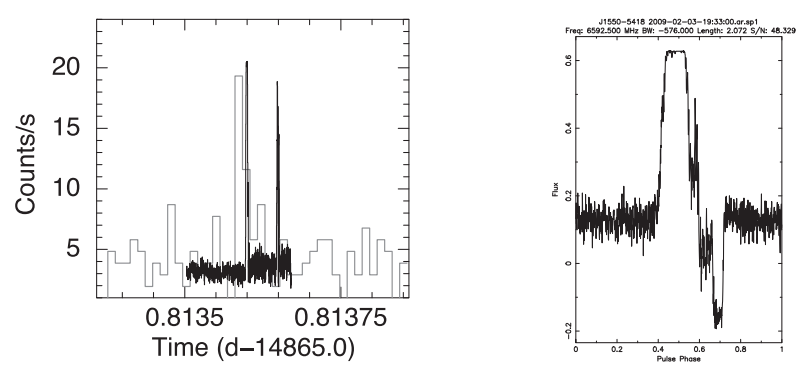

Figure 1. Left: 6-GHz radio (black line) and 1-10keV (grey) light-curves of J1550-5418 (February 2009); the correlation between the first (saturated) radio pulse and the X-ray short burst is evident. Right: the saturated radio pulse profile recorded in the AFB 1-bit sampled data.

with prepsubband, and single pulses with SNR $>7$ and width up to the maximum rebinning allowed (300 samples) were searched with the python script single_pulse_search. py. The de-dispersed time series were also searched for periodic signals. XMM data were processed using the SAS software package. Standard data screening criteria were applied in the extraction of scientific products.

\section{Results}

No single pulse of likely extragalactic origin was found in 16.2 total hours of data analysed. Given the coarse frequency and time resolution of most observations (whose main aim was to find a transient periodic signal from a long period radio magnetar) and the expected FRB rates, this is not surprising. Periodicity searches resulted in the detection of the known pulsar B1830-08 in the data of SGR J1833-0832 with a signal-tonoise ratio of 375 . J1809-1943 was detected with flux varying along the $7 \mathrm{hr}$ observation. In the case of J1550-5418 no persistent periodic signal was detected, while two single pulses (separated by just one radio silent rotation) were found at the pulsar's DM (Fig. 1). The first of the two pulses occurred at the same time as an X-ray burst, with a $0.02 \%$ probability for chance coincidence. This pulse was so strong as to saturate the instrument (AFB data 1-bit sampled every $1 \mathrm{~ms}$ over $1923-\mathrm{MHz}$ wide channels) for $0.2 \mathrm{~s}$, implying a lower limit on its flux of $1.1 \mathrm{Jy}$, and on its fluence of $200 \mathrm{Jy} \mathrm{ms}$.

\section{Discussion}

The simultaneous detection of a strong radio pulse and an X-ray burst from J1550-5418 is at odds with what seen in the high-B pulsar J1119-6127, where magnetar-like X-ray bursts quenched the radio emission (see R. Archibald et al., in this volume), implying a complex and varied interplay between X-ray and radio emission. In the framework of FRBs (Lorimer et al 2007, Thornton et al. 2013), the magnetar models are one of the favourite explanations for their emission; the simultaneous detection of a radio pulse and a magnetar X-ray burst could be important to validate these models. Because of pileup in the XMM data we could not get a reliable fluence for the burst. Since this burst looks unremarkable in X-rays, to give a crude estimate of the ratio $\eta_{J 1550}$ between radio and X-ray fluence we can, however, use the fluences measured by Scholz \& Kaspi (2011) for all the bursts recorded by Swift during the 2009 outburst, when also our observations were taken. With these numbers we get $\eta_{J 1550}>10^{9-11} \mathrm{Jy} \mathrm{ms} \mathrm{erg}^{-1} \mathrm{~cm}^{2}$, in agreement with the lower limits measured for 15 FRBs (Tendulkar, Kaspi \& Patel 2016), implying that the saturated radio pulse reported here could be a scaled down version of an FRB, and giving some support to magnetar models for these mysterious signals. 


\section{References}

Camilo F., Ransom S. M., Halpern, et al., 2006 Nature, 442, 892

Camilo F., Ransom S. M., Halpern J. P., \& Reynolds J. 2007, ApJ, 666L, 93

Lorimer D. R., Bailes M., McLaughlin M. A., et al., 2007, Science, 318, 777

Scholz P. \& Kaspi V. M., 2011 ApJ, 739, 94

Thornton D., Stappers B., Bailes M., et al., 2013 Science, 341, 53

Tendulkar S., Kaspi V. M., \& Patel C., 2016 ApJ, 827, 59 